\title{
EL EMPRENDEDOR SOCIAL Y SU ROL DENTRO DE LOS PROCESOS DE NEGOCIACION EN LOS CONFLICTOS ORGANIZACIONALES
}

\author{
Fredys Padilla González', \\ Nair Cantillo Campo², \\ Carlos Julio Pedraza Reyes ${ }^{3}$
}

Aceptado: 12 de agosto de 2013

\section{Resumen}

Los conflictos se conocen desde el comienzo de la humanidad y ha sido una constante en la vida personal como en la organizacional, esto motivo a ver la forma como miembros importantes de las compañías que tienen plasmado en su interior el sentido social fueran los canalizadores y fomentadores de las negociaciones, por esto la importancia de las dos variables en estudio. El objetivo de este artículo es analizar el emprendimiento social y su rol dentro de los procesos de negociación en los conflictos organizacionales. Para tal efecto se consultaron textos de autores como: Alonso y Galve (2008), Brown y Moberg (2007). El tipo de investigación fue descriptiva y documental, apoyado en un diseño bibliográfico. En líneas generales enunciaremos una de las principales conclusiones donde el producto de la revisión documental destaca que el emprendedor social es una pieza clave en el punto álgido de las negociaciones, por su capacidad social de liderazgo y por su visión y creatividad en los contextos organizacionales.

Palabras clave: Emprendedor, Emprendedor Social, Conflicto y Negociación

\section{THE SOCIAL ENTREPRENEUR AND ITS ROLE IN THE NEGOTIATION PROCESS IN ORGANIZATIONAL CONFLICT}

\section{Abstract}

A conflict are known from the beginning of mankind and has been a constant in life and in the organizational staff, this reason to see how important members of the companies that are embodied within the social sense were the channel and encourage of negotiations, hence the importance of the two variables under study. The aim of this paper is to analyze social entre-

\footnotetext{
1 Profesional en Contaduría Pública, Especialista en Educación superior a Distancia y Magister en Mercadeo, correo electrónico: fredys.padilla@unad.edu.co

${ }^{2}$ Administradora de empresas. Msc. Mercadeo. Doctora. En Ciencias gerenciales. Docente universitario, correo electrónico: nair.cantillo@unad.edu.co

${ }^{3}$ Profesional en Relaciones internacionales. Especialización Educación en Derechos Humanos. Msc. Gerencia de Mercadeo. Doctor En Ciencias Gerenciales. Cursos de alta gerencia, correo electrónico: carlosjpedrazar@hotmail.com
} 
preneurship and its role in the processes of negotiation in organizational conflicts. For this purpose texts were consulted as Alonso and Galve (2008), Brown and Moberg (2007). The type of research was descriptive and documentary, supported by a bibliographical design. Overall we state one of the main conclusions where the product of the literature review highlights that the social entrepreneur is a key player in the height of the negotiations, social ability and leadership for their vision and creativity in organizational settings.

Keywords: Entrepreneur, Social Entrepreneur, Conflict and Negotiation

\section{Introducción}

Las organizaciones como sistema abiertos, conformado por un talento humano con espíritu laboral, donde se encuentran insertas diversidad de géneros, razas, religiones y políticas, esta pluralidad ha ocasionado diferentes clases de conflictos internos y por ende diferentes clases de negociaciones, esto motivo a que surgieran los emprendedores sociales, los cuales con sus ideas innovadoras y trasformadora han provocado grandes cambios en las organizaciones.

Si bien es cierto este cambio ha sido visto por el común como ventaja y una nueva forma de ser rentable en los negocios, buscando un desarrollo que le permita promover organizaciones notables en lo social y en lo económico. Los emprendedores son personas que trabajando en una empresa han tenido la visión de los problemas en la parte social, dándole esto una experiencia para proponer ideas innovadoras para solucionar problemas sociales. Es así como se percibe la influencia del emprendedor dentro del buen funcionamiento del negocio, y en virtud de ello encontraremos actitudes y escenarios dispuesto a asumir nuevos retos.

Por otro lado, las organizaciones, como sistemas sociales abiertos, sufren continuamente conflictos internos y externos como consecuencia de las relaciones que se establecen entre los miembro que las componen y de las relaciones que la misma organización mantienen con sus similares, por esta razón es de importancia el conocimiento y manejo de las técnicas de negociación.

En este orden de ideas y siguiendo las pautas anteriores en este estudio se analizó el emprendedor social y su rol dentro de los procesos de negociación en los conflictos organizacionales.

El emprendedor social dentro de las organizaciones tomado como generador de cambio En los últimos años las organizaciones en el ámbito comercial, tecnológico e investigativo, a tribuido a la globalización de los mercados, aunado a ella el crecimiento de la población mundial, y con el fin de adaptarse a las exigencias del entorno para mantenerse actualizada bajo las perspectivas de competitividad, resultando fundamental para adaptarse a ello, el análisis de los problemas internos y externos.

En este sentido las organizaciones han empezado a tener una nueva visión de los negocios y están optando cambios en la parte social, se ha visto la importancia de iniciar proyectos empresariales que faciliten satisfacción, que cumpla con el fin de erigir organizaciones para forjar riqueza pero que a la vez no perjudique a la sociedad. Este cambio ha sido visto por el común como ventaja y una nueva forma de ser rentable en los negocios, buscando un desarrollo que le 
permita promover organizaciones notables en lo social, económico y territorial. Los emprendedores son personas que trabajando en una empresa han tenido la visión de los problemas en la parte social, dándole esto una experiencia para proponer ideas innovadoras para solucionar problemas sociales.

En virtud de ello, se ha vivido una constante introducción de conceptos claves que hace a las empresas distintivas convirtiéndose de una mera fuente tradicional en empresas contemporáneas, enmarcada en empresa del futuro, así como la anexión de técnicas gerenciales relacionadas con valores para gestionar los negocios. En tal sentido, la productividad como valor económico con capacidad de producir, vender más, a menor costo, con una buena rentabilidad y calidad excelente, como la de crecer en los mercados internos como externos.

\section{Los conflictos y su impacto en las organizaciones}

Las teorías nos permiten conocer el origen del debate y ordenar los principales conceptos en función de las distintas corrientes teóricas, en las que no solo encontraremos posturas en contraste, sino también un fructífero dialogo con relación al orden social, la integración y el cambio social. En las teorías de las relaciones laborales hablar de dos grandes miradas o perspectivas, la primera de carácter funcionalista, el consenso seria la característica dominante de las relaciones laborales, el conflicto es meramente coyuntural. Su origen es difuso, múltiple y no está vinculado necesariamente a la lucha de clases.

El sindicalismo se concibe como una institución de mediación entre los trabajadores, los empresarios y el Estado. La negociación colectiva se entiende como una institución de cooperación alternativa a la huelga, acentuando el equilibrio general como característica básica de los sistemas de relación laboral. La segunda mirada es una interpretación opuesta, la característica básica de la relación laboral es el conflicto, que tiene un origen estructural. Se relaciona con la desigualdad social, La asimetría de poder entre capital y trabajo, De alos y Martin (2002).

Las organizaciones, como sistemas sociales abiertos, sufren continuamente conflictos internos y externos como consecuencia de las relaciones que se establecen entre los miembro que las componen y de las relaciones que la misma organización mantienen con sus similares, por esta razón es de importancia el conocimiento y manejo de las técnicas de negociación.

\section{El emprendedor visto como soporte organizacional}

Las organizaciones en su carrera contra el tiempo han buscado soporte en personas o equipos que se encarguen de encontrar ideas y nuevas oportunidades que identifiquen mediante la innovación y la exploración de cambios nuevas formas de negocios, bienes o servicios que creen un valor adicional o diferente para sus clientes. Porque ellos a su vez son los encargados de evaluar, mirar, estudiar e identificar coyunturas viables teniendo como punta de mira el enfoque de los clientes, el desempeño de la calidad, como la integridad y la responsabilidad de la compañía.

En este sentido, Longenecker y otros (2009), manifiesta que los emprendedores son personas que identifican necesidades de mercado e inician nuevos negocios o empresas para satisfacerlas. Son personas que asumen riesgos y proveen el ímpetu para el cambio, la innovación y el progreso en la vida económica.

De igual forma, la Cámara de Comercio, Ministerio de Educación y Ciencia de España (2006), dice qué emprender es un sentido amplio, apunta a tomar decisiones con algún riesgo; en un sentido más restringido, a crear empresas, donde el riesgo es mayor. En ambos casos subyacen planteamientos económicos que la escuela puede despertar, alentar o fortalecer. 
Así mismo Castillo (2008), manifiesta que emprender va mucho más allá de crear una empresa, más bien implica crear riqueza, diseñando e implementando planes que incluyan innovación y creatividad.

La creatividad y la innovación no son suficientes para generar riquezas, de aquí surge la necesidad de crear un valor potencial como son los emprendedores que acondicione los esfuerzos enmarcándolos dentro de las políticas organizacionales llevándolos a cumplir las metas.

\section{El emprendedor social y su gestion social e innovadora}

Es así como durante algún tiempo se confundió al emprendedor social con el empresario y este con el capitalismo, olvidando que la productividad no es la única garantía para asegurar la permanencia en el mercado, pues bien en la actualidad se hace referencia a factores endógenos y el requerimiento axiológico para responder ante la sociedad mostrando su estabilidad como ventaja competitiva. Cuando se piensa en todo lo anterior viene a la memoria la importancia de tener o formar dentro de las organizaciones los emprendedores, personas innovadoras y con visión para llevar a cabo los proyectos no solo para atender las necesidades económicas, sino aquellas que faciliten responder ante un problema o inquietud social.

En este sentido Soto y Cárdenas (2007). Argumentan que el auténtico emprendedor social es aquel que acepta y lleva a cabo una transformación social o innovación, material o mental, sea un nuevo producto o un método de trabajo, una acción política o un valor social. Es evidente la importancia de la gestión del emprendedor en las empresas, que junto con la relevancia del emprendimiento social como eje fundamental en el renacer de las nuevas organizaciones, que están en busca de otros factores de índole social y cultural asociados a la orientación empresarial, los cuales permiten garantizar un desarrollo sostenible y sustentable.
Por tanto su repercusión tendrá lugar en el cumplimiento estratégico de su filosofía de gestión enmarcada en la práctica de valores éticos compartidos convirtiéndose en un modo de conducta organizacional. Que busca con el emprendimiento social darle un viraje humanitario a la organización, que sea vista no solamente como un ente económico, sino como un sujeto responsable con su entorno, donde la comunidad perciba la importancia que tiene para la compañía porque recoge las dudas, la problemática y los deseos, para que en trabajo conjunto dar una solución beneficiosa para las partes.

En virtud de ello, el emprendimiento social es un término de interés en las organizaciones actuales, representando la contribución activa en lo legal, ético y ambientalista, siendo argumentada por una conciencia empresarial orientada al desarrollo comunitario en la sociedad moderna, apoyada del sistema económico de cada país.

Por consiguiente, se debe establecer un convenio de las organizaciones con la comunidad, a fin de cumplir con sus fines, donde se busca generar una conciencia social que guíen a los sujetos como personas y como colectivos, buscando la socialización a través del sentido ético, en el cual además de formar hacia el alcance de un fin lucrativo, también en sus obligaciones como actor social clave para el desarrollo sostenible que lo legitima o certifica en su comportamiento con la comunidad, en términos ético- morales.

Al respecto, Austin (2006), señala el emprendimiento social comprende aquellas compañías en forma independiente o a través de alianzas con organismos gubernamentales y de la sociedad civil, puedan contribuir al proceso de mejora de la humanidad. Dentro de este contexto, las organizaciones deben prepararse constantemente junto con organismos gubernamentales o las ONG, para afrontar los retos predominantes del entorno social. 
Con el propósito de prever o anticiparse, a nuevos escenarios como base de sus responsabilidades u obligaciones comunitarias; sin embargo, hacerlo con sentido moralista, vinculando su plataforma ética en la práctica de la cultura organizacional, promoviendo el compromiso donde se formalice la participación ciudadana individual y colectiva.

De esta manera se podrá establecer la gestión del emprendimiento social como una estrategia organizacional tanto para la formación como para la educación de personas con capacidad de liderar una sociedad justa, humanista, por demás eficiente, logrando ser el engranaje del desarrollo económico, ambiental y social de su colectividad, para lograr y mantener el crecimiento sustentable de las comunidades.

\section{Habilidades del emprendedor social como causa de impacto en las negociaciones}

Los emprendedores sociales deben gozar de ciertas habilidades naturales que se deben manifestar en el trascurso de su vida laboral y social, dando pie a consolidarse como personas sobresalientes e integras capaces de enfocar y lograr desarrollos significativo en la organización.

Como lo publicado por el B.I.D (Banco Interamericano de Desarrollo) (2006), donde manifiesta que los emprendedores sociales deben tener ciertas habilidades, porque estas son las destrezas personales o profesionales que posee un individuo o grupo de trabajo para transformarlas en capacidades organizacionales. Vamos a conocer los distintos tipos de habilidades que debe de estar inmersas dentro de un emprendedor.

\section{Habilidad Emprendedora}

Consiste en la capacidad de un individuo para identificar y aprovechar oportunidades que le permitan iniciar un emprendimiento, independiente de los recursos que tenga.

\section{Habilidad de Diagnóstico}

La capacidad para estudiar con rigor y comprender el conjunto de causas y consecuencias en el corto, mediano y largo plazo de los problemas sociales que aquejan a la entorno, el líder diseña un plan para dar respuesta a un problema. La habilidad de diagnóstico comprende la articulación de una solución factible y efectiva a través de un modelo de cambio social.

\section{Habilidad para Armonizar}

Le permite al líder conciliar el foco estratégico del emprendedor social con las agendas de los diferentes grupos de interés. Esta alineación contribuye a que el emprendedor social sea dotado de estructuras, procesos y equipos de trabajo, para que sea concebido como un conjunto de acciones consecuentes con la misión y la estrategia de la organización.

\section{Habilidades para Motivar el equipo}

Con el objeto de alcanzar la institucionalización de los emprendedores sociales son necesarias la eficiencia y efectividad; para lograrlos es importante conformar un equipo de trabajo comprometido e identificado con la causa social. La habilidad de los líderes para motivar es uno de los componentes más relevantes en la consecución de estos objetivos.

\section{Habilidad para Delegar}

Es la capacidad de los líderes centrales para asignar a otros unas funciones, convirtiéndolos en sus mentores y atribuyéndole poderes para que asuman la responsabilidad de la operación de las unidades autónomas.

\section{Habilidad Visionaria y de Compromiso Social} La visión social del líder se define como la capacidad que le permite comprender la problemática social de su entorno desde una perspectiva solidaria, e identificarse con la realidad que enfrentan distintos grupos de la sociedad. 
El compromiso social del líder se observa a través de su participación en el emprendimiento mismo y en la sensibilidad que muestra para con su personal y el respeto por el ser humano sin importar su estrato social.

\section{Habilidad Política y Negociadora}

En un conglomerado social, es común que los líderes de la organización desplieguen una amplia habilidad política y negociadora para canalizar los objetivos contrapuestos, establecer relaciones efectivas con distintos grupos de interés y consolidar la visibilidad del emprendedor social a través de estas habilidades de liderazgo logran aprovechar las sinergias entre las distintas actividades y disminuir las tensiones que puedan surgir por los conflictos de interés entre las unidades.

\section{El conflicto y su comportamiento en la sociedad}

Thomas (1976), lo considera como un proceso que se origina cuando una persona percibe que otra ha frustrado o está a punto de impedir el logro de algunos de sus objetivos o intereses.

Hay conflicto cuando determinados actores defienden valores opuestos, o bien persiguen metas opuestas, en forma simultánea o competitiva el mismo objetivo. La competencia es la principal fuente de conflicto y lo más frecuente son los conflictos de intereses o la carrera o persecución de metas diferentes.

La competencia se da porque las organizaciones están basadas en la división del trabajo, lo que da por resultado una diferenciación del trabajo en áreas funcionales, así como una diferenciación en roles. Dado esto, se presentan intereses y objetivos distintos.

El conflicto en las organizaciones puede ser visto como:

A. Algo disfuncional y negativo que debe evitarse, ya que:
- se desvían los esfuerzos del trabajo diario y de los objetivos,

- pérdida de tiempo al dedicarse a resolver el conflicto,

- grandes costos a la organización,

- problemas psicológicos en las personas,

- deterioro del clima social del grupo.

B. Algo funcional y beneficioso para los individuos y la organización. El conflicto puede:

- aumentar la motivación y energía disponibles para realizar tareas requeridas por el sistema,

- incrementar la capacidad de innovación de los individuos y del sistema a causa de una mayor diversidad de puntos de vista,

- cada persona puede adquirir una comprensión mayor de su posición, ya que el conflicto los fuerza a expresar sus puntos de vista y manifestar todos los argumentos en que se apoya,

- cada parte puede lograr mayor conciencia de su propia identidad al incrementar la cohesión del grupo y su desempeño,

- mide el poder o la habilidad. La coerción y el control requieren una superioridad de poder de una de las partes, mientras que la solución de problemas requiere un equilibrio de poder entre las partes.

Que el conflicto sea positivo o negativo dependerá de cómo se maneje. En general las discrepancias son útiles para exponer las debilidades organizacionales, pero deben manejarse en forma rápida antes de que se salgan de las manos.

Para impedir que el manejo del conflicto llegue a la etapa sentida, lo ideal es que el gerente intervenga en la etapa latente y actué sobre las fuentes del conflicto cuidando eso sí que el cambio efectivamente se provoque. En toda organización el conflicto es inevitable e incide significativamente en el comportamiento organizacional y desde luego, según la forma como se manipule, los resultados pueden ser desastrosos, bien conducir a la organización a su eficiencia. 
Es por eso la relevancia de que la supervisión, el jefe entre otros, logren que la administración del conflicto constituya una parte fundamental del trabajo y de su verdadero estilo de liderazgo. En cuanto a la naturaleza y sus causas existen muchísimas aportaciones de acuerdo a los investigadores. Así algunos concluyen señalando que para definir la naturaleza del conflicto es preciso alcanzar los propios objetivos, aunque a veces puede ser muy difícil, debido a que nuestros objetivos suelen ir más allá del problema que se manifiesta en un momento dado.

Sin embargo, hay quienes señalan, que las diferentes creencias o sistemas de valores pueden dar lugar a conflictos cuando las personas que albergan estas diferencias tan fundamentales compiten por objetivos diferentes de los cuáles perciben que sólo podrán alcanzarse uno.

\section{Principales causas y tipos de conflictos en las organizaciones \\ Brown y Moberg 2007señalan tres tipos:}

Conflictos de papel. Los conflictos de papel aparecen cuando un individuo experimenta desacuerdo sobre lo que se espera que haga.

Conflicto interpersonal. Cuando surge entre dos o más individuos independientemente de las presiones del papel y las formas más comunes son: 1.- conflictos por recursos escasos, estos son aquellos sobre los presupuestos y las instalaciones. Son difíciles de manejar cuando la escasez es absoluta y cuando no pueden compartirse los recursos. 2.- por objetivos, que surgen cuando hay desacuerdo sobre las metas, objetivos o valores. 3.- Por los medios, que son más intensos cuando hay poco conocimiento u opinión experta asequible que ayude a identificar los medios apropiados. 4.- Por los hechos. Dos ingenieros que trabajan en forma independiente pueden salir con diferentes respuestas al mismo problema de diseño del producto. Muchos conflictos por los hechos pueden resolverse mediante una mejor comunicación y una corriente de información más eficaz.

Conflictos entre unidades. Ocurre entre los grupos de una organización, como los departamentos, secciones o equipos de trabajo. Las áreas específicas de desacuerdo entre estos grupos pueden ser las mismas que se dan en el conflicto interpersonal, pero las causas por lo general son diferentes.

El conflicto se percibe como destructivo cuando:

- Distrae la atención de los temas importantes,

- Sabotea la moral, la auto-imagen o la identidad grupal

- Polariza a las personas, y a los grupos, destruyendo la cooperación,

- Confronta a los empleados y los estimula hacia conductas dañinas e irresponsables, incluyendo insultos, agresiones físicas, tácticas de exclusión y hostigamiento a otros,

- Genera percepciones individuales de exclusión, hostilidad y humillación que podrían volver como actos armados de revancha contra la institución o empleados «agresores».

Una evaluación de los costos del conflicto envuelve estimar diferentes aspectos, tales como: Excesiva rotación del personal, Productividad que se reduce, Problemas con la calidad del producto, Plazos que se alargan o se pierden, mayores costos de supervisión de empleados, La cooperación entre grupos disminuye, Conductas de abuso y hostigamiento sin control, Pérdida de prestigio y poder por la gerencia y más insatisfacción de parte de los clientes

\section{Como se logra la coordinación}

- Coordinación y Rutinas

- Cuando los problemas se repiten y son de rutina, puede realizarse la coordinación planeada. Aquí se puede efectuar la coordinación mediante programas preestablecidos, que especifican qué actividades se van a ejecutar 
y en qué momento. En cambio en las situaciones que están cambiando rápidamente y en las cuales se están presentando siempre nuevos y diversos problemas, para este caso puede ser más adecuada la coordinación por retroalimentación. En ésta se crea en el sistema de coordinación la capacidad específica de percibir desviaciones e informar a los interesados.

- Coordinación Jerárquica. Tal vez la forma más familiar de buscar coordinación es atenerse a la organización jerárquica formal. La coordinación jerárquica puede tomar muchas formas, desde la muy sencilla de que sea un ejecutivo central, como el presidente, el que hace el seguimiento y enlace de las actividades de los vicepresidentes de ventas y de producción. Un ejemplo es, en un estudio de toma de decisiones gerenciales en varias sociedades anónimas de las más grandes, se encontró que el jefe ejecutivo era casi siempre el árbitro final cuando se presentaban puntos de vista distintos entre los vicepresidentes.

- vintegración Estructural. Se han usado diversos integradores especiales para actuar como mediadores de conflictos potenciales y facilitar la coordinación. Esto no es una idea nueva; inclusive Fayol, quien recomendó una conferencia semanal de los jefes de departamentos para facilitar la coordinación a la luz de los hechos corrientes. Si esto no era posible, aconsejaba el uso de empleados especiales como funcionarios de enlace.

\section{Gerenciamiento del conflicto de un modo productivo}

El conflicto bien procesado es la manera más usual en la cual las organizaciones procesan su cambio a nivel institucional. De este modo se podría caracterizar al conflicto como constructivo cuando:

- Resulta en la clarificación de temas importantes

- Resulta en soluciones para algunos problemas y mantiene el proceso del cambio moviéndose hacia adelante
- Envuelve a las partes en la solución de sus propios problemas

- Produce comunicación auténtica

- Ayuda a liberar las emociones y el stress

- Promueve cooperación entre personas que aprenden más sobre cada una mientras trabajan para resolver su conflicto

- Las personas aprenden las técnicas necesarias, para manejar futuros conflictos.

\section{Necesidad de gerenciar los conflictos}

Las organizaciones delegan en los supervisores la responsabilidad por el manejo de los conflictos, de un modo casi automático, y sin averiguar cuáles son las capacidades que los supervisores tienen.

A veces tienen sus propios intereses incluidos en el conflicto, lo cual puede aumentar la frustración y el resentimiento de los trabajadores en conflicto. El hecho de que los gerentes sean Ilamados a hacer este trabajo, con consecuencias serias si fracasan, ilumina la necesidad de incluir capacitación en habilidades de manejo de conflictos en el lugar de trabajo.

De acuerdo con este punto, en corporaciones con y sin sindicatos, en agencias del gobierno, instituciones académicas, organizaciones profesionales y de servicios, escuela y hospitales, los empleados y los empleadores están ahora repensando la naturaleza de los sistemas de gerenciamiento de conflictos y revisando los canales establecidos desde antes para resolver sus propias diferencias.

\section{Estilos gerenciales de manejo de conflictos}

Los gerentes o supervisores usan estilos de conflictos que ya vienen dentro de su personalidad, aprendidos desde largo tiempo atrás (Cropanzano et al, 1999). Veamos algunos de estos estilos:

- Evitación: una táctica muy común que se usa en más del $20 \%$ de los casos, es ignorar el problema. El supervisor ignora sus deberes y hace 
que no ve la disputa, y de este modo, niega también que se pueda intervenir.

- Aconsejar: «aconsejar» es fácil, porque lo único que hay que hacer es invitar a los dos lados a sentarse a la misma mesa. El supervisor está allí para invitarlos a hablar, pero no provee ninguna facilitación del proceso; o sea que si las partes no quieren hablar, no hay nada que hacer. Se les puede predicar paz y comprensión, pero finalmente harán lo que deseen hacer, y si eso es seguir compitiendo, pues así lo harán. Este método se usa en más de un $30 \%$ de casos

- Control autocrático: El gerente puede actuar de modo directivo y hacer preguntas desde su autoridad. Esto no es conductivo a una mayor cooperación de los empleados, que se ven frente a un juez que dirige la conversación, elige los temas a tratar, y recomienda una solución que le conviene a él solamente. Usado en un $20 \%$ de casos.

- Hacer amenazas. Los individuos pueden ser invitados a una conversación donde se los instiga a hacer las paces a riesgo de sufrir otras consecuencias. Se ven obligados a abandonar sus reclamos rápidamente para restablecer la paz, sin tener oportunidad de explicar que les pasa y que problemas tienen. Usado en un $15 \%$ de casos

- Funcionar como árbitro. El gerente invita a las partes, les hace contar sus historias y después decide por su cuenta cual es la solución, y la impone a los subordinados. En este método por lo menos pueden explicar lo que les pasa, pero se ven obligados a aceptar una solución en la cual no han participado, que viene desde arriba. Hay algunas culturas de tipo patriarcal que ven este estilo como el más adecuado, y las partes aceptan la solución gustosamente. Usado en un $15 \%$ de los casos.
Si para los trabajadores el actuar una disputa en público es la manera preferida de hacer saber que existen diferencias serias, se entiende que cuando el gerente entra en la disputa, ya sea para mediar o tomar una decisión, está haciendo un puente para la diferencia. ¿Cuan efectiva es esta intervención? Las respuestas del tipo aconsejar son las más preferidas, seguidas por estilos autocráticos y adversariales, mientras los incentivos negativos y la evitación son vistas como las tácticas menos efectivas, de acuerdo a Shapiro y Rosen (1994). Ambos carecen de un elemento vital, como es el incluir a los trabajadores de modo que trabajen más cooperativamente en el futuro.

\section{Aplicación del coaching ejecutivo para refor- zar las habilidades del gerente}

A través de proveerles un coach de conflictos personal se intenta mejorar el desempeño de los gerentes solucionando divergencias, a través de motivarlos, darles información, escucharlos y ofrecerles ideas nuevas.

Un coach también les enseña nuevas habilidades que los gerentes pueden demostrar a sus subordinados, convirtiéndose en modelo de rol para ellos como líderes. La elección de un coach que les ayude a mejorar su rendimiento es a veces la primera decisión que se hace en el camino de aprender cómo liderar en organizaciones conflictivas.

\section{La inclusión de la mediacion gerencial}

Junto con la incorporación de un coach, la implementación de la mediación es una buena propuesta en el lugar de trabajo. La mediación refuerza a los trabajadores en la búsqueda de la mejor solución para sus propias diferencias, al incluirlos a ellos dentro del diseño e implementación de procesos de mediación.

De este modo se promueve el compromiso y la responsabilidad desde los rangos inferiores 
hacia arriba para que las cosas se hagan, mientras al mismo tiempo se le da al gerente una visión profunda de como los trabajadores perciben sus propias circunstancias, y donde están las líneas de fractura. De este modo, el gerente podría evitar conflictos nuevos en el futuro, usando la información que ahora se le presenta.

El desarrollo de los sistemas de resolución de conflictos hoy en día muestra intervenciones diseñadas con la óptica de enfocar la organización en su totalidad. Aquí se incluyen prácticas tales como:

- La institución del ombudsman, una entidad que tiene la función de recolectar información sobre las quejas de los empleados, agregarlos en documentos que reflejen distintas categorías de quejas y la identificación de aquellas soluciones sistémicas que ofrecerían soluciones a las mismas. Esta función no está pensada para ofrecer soluciones individuales, pero si para buscar patrones y regularidades en las quejas que revelen las deficiencias sistémicas a resolver por medio de cambios en las políticas organizacionales.

\section{- Los sistemas diseñados para toda la or-} ganización, que les ofrece a los empleados maneras sistemáticas de recolectar, procesar, solucionar entredichos y quejas, están basados en una serie de valores centrales previamente identificados con la disciplina de desarrollo organizacional. La aceptación de la diversidad, la transparencia, el reconocimiento de las diferencias y el aprender de ellas, la apertura de conceptos e ideas, y la búsqueda de la continua retroalimentación, son críticos. Aquí el éxito se mide por el grado de participación en los procesos de cambio, y por el grado del compromiso en los cambios propuestos.

Los sistemas de gerenciamiento de conflictos en organizaciones son una nueva herramienta, que usa métodos ya probados como la mediación, para promover la participación de los empleados en procesos de decisiones para identificar, mejorar y cambiar las áreas problemáticas de su organización. De este modo, la participación de los empleados no solo en la generación pero en la identificación y solución de las disputas organizacionales es el elemento básico de un lugar de trabajo más productivo y eficaz.

\section{Conclusión}

Es pertinente señalar que el emprendedor social debe gozar de ciertas habilidades naturales que se deben manifestar en el trascurso de su vida laboral y social, dando pie a consolidarse como personas sobresaliente e integras capaces de enfocar y lograr desarrollos significativos en la organización. Por tanto su repercusión tendrá lugar en el cumplimiento estratégico de su filosofía de gestión enmarcada en la práctica de valores éticos compartidos convirtiéndose en un modo de conducta organizacional. En este orden de ideas el emprendimiento social darle un viraje humanitario a la organización, que sea vista no solamente como un ente económico, sino como un sujeto responsable con su entorno, donde la comunidad perciba la importancia que tiene para la compañía porque recoge las dudas, la problemática y los deseos, para que en trabajo conjunto dar una solución beneficiosa para las partes.

En este sentido es común que los líderes de la organización desplieguen una amplia habilidad política y negociadora para canalizar los objetivos contrapuestos, establecer relaciones efectivas con distintos grupos de interés y consolidar la visibilidad del emprendedor social a través de estas habilidades de liderazgo logran aprovechar las sinergias entre las distintas actividades y disminuir las tensiones que puedan surgir por los conflictos de interés entre las unidades.

Los emprendedores desempeñan una función trascendental en la resolución de conflicto bien 
procesado es la manera más usual en la cual las organizaciones procesan su cambio a nivel institucional. De este modo se podría caracterizar al conflicto como constructivo cuando.

\section{Referencias}

Arias, F (2006). El Proyecto de Investigación. Caracas: Editorial Episteme.

Alonso María y Galve Carmen (2008), El emprendedor y La Empresa. Zaragoza: Editorial Acciones e Investigaciones Sociales.

Austin, J. et al. (2006). Gestión efectiva de emprendimiento social. Washington. Editorial Banco Interamericano

Banco Interamericano de Desarrollo (B.I.D) (2006), Gestión Efectiva de Emprendimientos Sociales. Bogotá: BID.

Cámara de Comercio y Ministerio de Educación y Ciencia (2006), Fomento del Espíritu Emprendedor en la Escuela. España. Imprenta. Modelo Madrid

Dees, JG. (1998). Sin fines de lucro emprendedor. Harvard Business Review, 76 (1): 54-67.

Dees, JG, Anderson, B. y Wei-Skillern, J (2004) Escala de impacto social: estrategias para la difusión de la innovación social. Stanford Social Innovation Review, 1 (4): 24-32.

Fernandez-Ruiz (2006). Los Jóvenes y la Creación de Empresas. Madrid. Consejo Superior de Investigaciones Científicas, Catalogo General de Publicaciones Oficiales
Hurtado, J (2007). Metodología de la Investigación. Caracas: Ediciones de la fundación Servicios y proyecciones para América Latina

Koontz, H y Weirich, H. (2004), Administración una perspectiva Global. México: Editorial Mac Graw Hill.

Longenecker, J., -Moore, G., Palich, C., Petty, L. (2009). Administración de Pequeñas Empresas. México: Editorial, Cengage Learning. Decima cuarta edición.

Méndez, C. (2006). Metodología Diseño y Desarrollo del Proceso de Investigación. Bogot\{a: Editorial Limusa. Cuarta Edición.

Morales, J. y S. Jiménez. (1999). El Grupo y sus Conflictos. Ciudad Real: Universidad de Castilla La Mancha.

Robbins, S. (2004). Comportamiento Organizacional. México: Editorial Pearson Pretice Hall. Décima edición.

Ríos, JN. (2003). Cómo Negociar a Partir de la Importancia del otro. Caracas :.Editorial Planeta

Soler, J. (2002). La Calidad y Eficiencia de las Negociaciones. Formas Estratégicas de la Negociación. Caracas: Publicaciones Universidad Católica Andrés Bello,

Soto, E, y Cárdenas, J. (2007) Ética En Las Organizaciones. México: Editorial. Mc Grac Hill Internacional.

Villasmil, H. (2007) Relaciones Laborales en Tiempos Difíciles. Caracas: Editorial Texto C.A - Universidad Católica Andrés Bello . 